\title{
Optimum Transmission Ranges in a Direct-Sequence Spread-Spectrum Multihop Packet Radio Network
}

\author{
ELVINO S. SOUSA, MEMber, IFEe, AND JOHN A. SILVESTER, SENIOR MFmbFr, IFEF
}

\begin{abstract}
In this paper, we obtain the optimum transmission ranges to maximize throughput for a direct-sequence spread-spectrum multihop packet radio network. In the analysis, we model the network selfinterference as a random variable which is equal to the sum of the interference power of all other terminals plus background noise. The model is applicable to other spread spectrum schemes where the interference of one user appears as a noise source with constant power spectral density to the other users. The network terminals are modeled as a random Poisson field of interference power emitters. The statistics of the interference power at a receiving terminal are obtained and show to be the stable distributions of a parameter that is dependent on the propagation power loss law. The optimum transmission range in such a network is of the form $C K^{4 x}$ where $C$ is a constant, $K$ is a function of the processing gain, the background noise power spectral density, and the degree of error-correction coding used, and $\alpha$ is related to the power loss law. The results obtained can be used in heuristics to determine optimum routing strategies in multihop networks.
\end{abstract}

\section{INTRODUCTION}

$I^{2}$ $\mathrm{N}$ a large distributed packet radio network, it is not always desirable for a terminal with a packet to send to attempt to transmit directly to the destination. It may be the case that the destination terminal is out of the transmitter's range, in which case it is impossible to transmit directly to the destination, or that the destination is within range, but the transmission protocol dictates that the packet take a series of short hops so as to achieve "space reuse" [1]-[3] which results in a higher network throughput. If a packet is transmitted directly to the destination, then there is no "store and forward" delay, but due to a larger number of potentially interfering terminals, the probability of a successful transmission is smaller than that for short-hop transmission. Using a model where terminals are assumed to be randomly distributed on the plane, Kleinrock and Silvester [1]-[2] were able to show that, to maximize overall network throughput, a terminal should transmit with a power so that the average number of terminals within range is six. Subsequent refinements to this analysis [4]-[5] which also include different rout-

Manuscript received February 10, 1989; revised November 3, 1989 This work was supported in part by the U.S. Army Research Office under Contract DAAG29-84-K-0084 and in part by the Natural Sciences and Engineering Research Council of Canada under a URF Grant. This paper was presented in part at the IEEE Military Communications Conference, Boston, MA October 1985

E. S. Sousa is with the Department of Electrical Engineering. Univer sity of Toronto, Toronto, Ont., Canada M5S 1A4.

J. A. Silvester is with the Communication Sciences Institute, Depart ment of Electrical Engineering-Systems, University of Southern California, Los Angeles, CA 90089.

IEEE Log Number 9035804 ing strategies have resulted in the conclusion that the number of neighbors of a terminal should be approximately six-eight.

These results were derived for narrow-band radio networks where at most one successful transmission at a time can occur in a given region of space. With spread spectrum signaling [6], multiple simultaneous successful transmissions are possible, and the above results do not apply. Moreover, the model used in these analyses assumes that the reception of a packet is independent of the distance from the transmitter to the receiver, as long as this distance is less than a critical radius. Also, if another terminal is undergoing a reception just outside of the transmission range of node $\boldsymbol{X}$, then according to these models, the reception is unaffected by the interference from a transmission by node $\boldsymbol{X}$. In this paper, we are concerned with the solution of the above problem (i.e., determining the optimum transmission ranges) for the direct sequence spread spectrum (DSSS) case. In the work reported in [1]-[5], the main issue is the choice of a transmitting power which defines a transmission radius that depends on the background noise. The choice of transmission power is a compromise between network connectivity and network interference which occurs in the form of packet collisions from terminals transmitting within the receiving radius. In such a model, the probability of packet success is assumed not to be a strong function of the distance between the transmitter and receiver if this distance is less than the transmission radius. In the case of spread spectrum transmission, a receiver will pick up some noise from each transmitter, and the packet error probability will be strongly dependent on the signal strength, even within the transmission radius. If the source of interference is mostly from other users, the probability of packet success will not depend as much on the absolute transmission power that each terminal uses since scaling the signal power also scales the interference by the same amount, but more on the transmission range selection. The transmission range should be expressed relative to the average distance between terminals. A related measure is the average number of terminals that are closer to the receiver than the transmitter. The transmission range must, of course, be less than the link distance or transmission radius that is defined by the transmitter power used. In general, the greater the transmission range, the lower is the probability of the transmission being successful. If the objective function is expected forward 
progress per transmission, then there is an optimum distance that the packet should attempt to travel per hop, and this distance is not necessarily the full distance to the destination. We assume that the transmitted power and processing gain are fixed, and that the main source of interference is multiaccess interference, and we find the expected forward progress per transmission, defined as the product of the probability of success times the link distance, in terms of the expected number of interferers that are closer to the receiver than the transmitter.

We derive the statistics of the received interference power at a terminal for a class of signal propagation laws (i.e., how the strength of a propagating signal varies with distance). For the inverse fourth power law (commonly used for ground radio), we show that the optimum transmission range is such that on the average, the number of terminals closer to the transmitter than the receiver is proportional to the square root of the processing gain.' Even though the analysis presented in this paper is for the direct sequence form of spread spectrum, depending on the detection scheme, the methdology may also be applicable to other spread spectrum schemes.

It is hoped that the technique presented here to analyze the interference at a terminal will have wider ranging implications in the analysis of routing strategies, adaptive techniques involving the variation of transmitter power, and the impact of jammers whose positions are randomly varying in space [7].

\section{Multihop Networks}

In most multihop network models employed by researchers thus far, each transmitter is assumed to use the same transmitting power. This power is assumed to determine a circle such that each terminal lying within the circle hears the given transmission, and any terminal lying outside the circle is completely unaffected by the transmission. If we denote the strength of the transmitted signal as a function of the distance from the transmitter by $g(r)$ (where $g$ stands for gain), then the above model corresponds to a $g$ of the form

$$
g(r)= \begin{cases}c & 0 \leq r \leq r_{0} \\ 0 & r>r_{0}\end{cases}
$$

where $c$ is some constant and $r_{0}$ is the critical radius determined by the transmitted power. With this model, the network can be represented by a graph with vertices corresponding to the terminals and an edge present between two vertices if and only if the distance between them is less than $r_{0}$. According to the model, a transmitted packet is successful if and only if no other terminal adjacent to the destination transmits at the same time.

A weakness of the above model can be illustrated with the aid of Fig. 1 which depicts a network of four terminals

\footnotetext{
'The processing gain is equal to the ratio of the system bandwidth to the uncoded data rate (see 16, p. 138]).
}

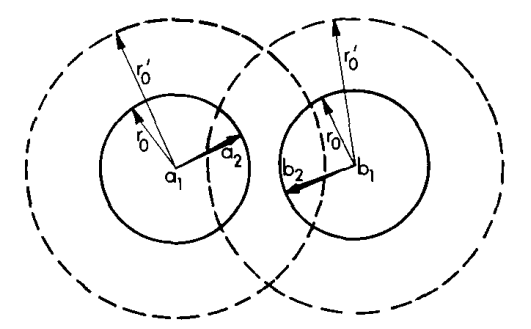

Fig. 1. The effect of transmission radius on interference.

that use a constant (equal) power to communicate. If the transmitting power is such that the critical radius is determined to be $r_{0}$, then according to the model, terminals $a_{1}$ and $\boldsymbol{b}_{1}$ can successfully transmit to terminals $\boldsymbol{a}_{2}$ and $\boldsymbol{b}_{2}$, respectively, at the same time. However, if the powers are increased so that the critical radius becomes $r_{0}^{\prime}$, then the above two communications cannot occur simultaneously. On the other hand, from a communication theory point of view, we know that the important parameter determining the success of a transmission is the signal-tonoise ratio at the receiver, which is not strongly dependent on the transmission power as long as all terminals transmit with the same power and the background noise is much smaller than the interference power.

The main drawback to the above model is that it does not discriminate between the differences in distance to a receiver of two transmitting terminals as long as the terminals are within the critical radius. An improved model is the capture model studied in [8]-[10], which is suited to $\mathrm{FM}$ transmission. If a given transmitting terminal, at distance $r_{1}$ from the receiver, is the closest transmitting terminal to the receiver, and if the next closest transmitting terminal is at a distance $r_{2}$, then the given transmission will be successful if $r_{1}<r_{0}$ and the ratio $r_{2} / r_{1}$ exceeds a threshold called the capture ratio.

The above models have been used for the nonspread spectrum case. A straightforward extension of these models to spread spectrum would result by setting a threshold on the maximum possible number of successful simultaneous transmissions and declaring that any time the number of transmissions exceeds the threshold, all transmissions are lost. However, since the powers of the various interferers vary greatly due to differences in their distances to the receiver, the number of transmitting terminals is not a good variable to work with in accounting for network interference at a particular receiver. This is especially the case with DSSS signaling. The analysis presented in this paper is an enhancement of that in [11] where we use the sum of the interference powers to model multiuser interference. This approach to interference modeling has been used in many analyses of cellular radio systems (e.g., [12]), spread spectrum multiple-access systems (e.g., [13]-[14]), and has recently also been adopted for throughput analysis of packet radio networks with fixed topologies and prespecified routing schemes in [15]. 


\section{System Model}

We assume a multihop packet radio network operating under heavy traffic conditions. The system is slotted, and in each slot, a terminal transmits a packet with probability $p$. The slot duration is assumed to be sufficiently large so as to allow a preamble for spreading code and carrier synchronization. The traffic matrix is assumed to be uniform. We are interested in calculating performance over many different changing topologies rather than for a specific terminal configuration. As a result, we obtain statistical performance values over a set of topologies. To do this, we model the positions of the terminals as a Poisson point process in the plane with parameter $\lambda$. If $A$ is the area of a given region $\boldsymbol{R}$ in the plane, then the probability of finding $k$ terminals in $R$ is given by

$$
P[k \text { in } R]=\frac{e^{-\lambda A}(\lambda A)^{k}}{k !}
$$

where $\lambda$ is the average number of terminals per unit area. We assume that during each slot, the network topology is constant; hence, the interference level will be constant over a packet transmission time. We also assume that the interference is independent from slot to slot. This is the assumption that was made in [1]-[2] and other work that followed. With this assumption, we may apply the results to a network with dynamically changing topology or to obtain average performance results for a collection of random networks. In this work, we are interested in obtaining optimum transmission ranges; hence, we assume a prespecified link in the network with a given distance $R$. The objective is to optimize the expected forward progress for a packet transmission as a function of the distance of the link in the presence of unknown network interferers that are modeled as a Poisson process. This philosophy is also consistent with the approach taken in [13] where a centralized system is analyzed.

\section{Interference Modeling}

The collision model for channel interference is not applicable in the case of DSSS signaling or when interfering signals have small powers. An alternative model that is not usually used in the narrow-band packet radio network literature, but has been used in many analysis of DSSS systems such as in [12]-[14] is that of summing the interference powers and treating the total interference as Gaussian noise. At the network analysis level, many spread spectrum schemes may be modeled this way. In this paper, we assume a direct sequence scheme with binary phase shift keying (DS/BPSK), although many other schemes such as DS/DPSK and the DPSK schemes that have been considered for cellular radio (e.g., [12]) allow the same type of analysis.

From a network analysis viewpoint, we are usually interested in calculating the probability of packet success given that the receiver is idle. This calculation is usually conditioned on some state of the network, and a tractable state model cannot usually contain much more than infor- mation on which terminals are transmitting and what are their received powers. A desirable model to work with in spread spectrum is the threshold model where we assume that the packet is successful if the signal to interference power ratio is greater than some threshold. To apply the threshold model, the variance of the interference at the detector must be directly related to the received interference power. In DS/BPSK systems with a large processing gain, it can indeed be shown that the noise at the detector due to one interferer is approximately Gaussian; however, the variance depends on the relative chip phase of the signal to the interferer. For the case of many interfering signals with approximately equal levels of interference, the chip phases average out and the noise variance is constant for a prespecified location of interferers. However, in some DS/BPSK system models with one strong interferer, the variance of the noise at the detector is a random variable that depends on the chip phase of the signal relative to the interferer. For a given total received power, the noise at the detector will have a variance that will vary from packet to packet. This variation can, however, be made small if there is an offset between the clocks of the various signals. We will assume this to be the case in this paper.

Threshold models in digital communications are ultimately dependent on the degree of error-correction coding. If the interference over a packet can be modeled as Gaussian noise, then the probability of packet success as a function of the signal-to-interference ratio is a smooth curve with a slope in the transition region that depends on the degree of error correction; for good long codes, the curve approaches a step function. In this paper, we model the actual smooth curve.

To calculate the packet success probability, we assume that the level of interference is constant over the transmission of a packet. The noise at the detector is due to interference from other users and to a constant background noise with power spectral density $N_{0} / 2$. We denote the symbol energy-to-noise ratio at the detector by $E_{b} / N_{0 \text { eff }}$ where $N_{0 \text { eff }} / 2$ is an equivalent white noise power spectral density for the same SNR at the detector. If the received signal has power $P_{0}$ and the interferers have powers $P_{1}, P_{2}, \cdots, P_{q}$, the average symbol energy-tonoise ratio at the detector in the case of DS/BPSK with rectangular chip pulse is then (see [16, eq. (17)] for a similar result with equal powers)

$$
\mu \triangleq \frac{E_{b}}{N_{\text {eff }}}=\left(\frac{2 Y}{3 L P_{0}}+\frac{1}{\mu_{0}}\right)^{-1}
$$

where $L$ is the processing gain, $Y=\Sigma_{i=1}^{q} P_{i}$, and $\mu_{0}=$ $E_{b} / N_{0}$. The parameter $\mu_{0}$ is the SNR at the detector in the absence of interferers.

For a given $\mu$, the probability of symbol error is

$$
p_{e}=\frac{1}{2} \operatorname{erfc}(\sqrt{\mu}) \text {. }
$$

The probability of packet success is dependent on the coding scheme. We denote the probability of packet success 
conditioned on the SNR $\mu$ as $s(\mu)$. As an example, for a $t$-error-correcting block code of length $n$, and under our assumption that symbol errors are independent given $\mu$, we have

$$
\begin{aligned}
& P[\text { success } / \mu] \triangleq s(\mu) \\
&=\sum_{i=0}^{t}\left(\begin{array}{l}
n \\
i
\end{array}\right)\left(\frac{1}{2} \operatorname{erfc} \sqrt{\mu}\right)^{i}\left(1-\frac{1}{2} \operatorname{erfc} \sqrt{\mu}\right)^{n-i}
\end{aligned}
$$

From packet to packet, the parameter $\mu$ is a random variable with density function $f_{\mu}(\cdot)$. The unconditional probability of packet success can then be written as

$$
\begin{aligned}
P_{s} & =\int_{0}^{\infty} s(x) f_{\mu}(x) d x \\
& =\int_{0}^{\infty}\left[1-F_{\mu}(x)\right] s^{\prime}(x) d x
\end{aligned}
$$

where the second expression is obtained after an integration by parts and $F_{\mu}(\cdot)$ is the probability distribution function of $\mu$.

\section{Interference at a Given Terminal}

To evaluate the above probability of packet success, we need to obtain the probability density function $f_{\mu}(\cdot)$. We prefer, though, to obtain the probability density $f_{Y}(\cdot)$ first, i.e., the probability density function for the multiuser interference power. Towards this end, we may assume that the terminal at which we are interested in obtaining the interference power is located at the origin.

Let $g(r)$ be the power of a given signal at a distance $r$ from the transmitter of the signal. In general, the exact form of $g$ will depend on the environment; however, it will always be very large for small $r$, and will approach zero as $r \rightarrow \infty$. For the moment, though, so as not to restrict ourselves to any particular environment, we merely assume that $g(r)$ satisfies the following two conditions:

1) $g(r)$ is monotone decreasing, $\lim _{r \rightarrow 0} g(r)=\infty$,

$$
\begin{aligned}
& \text { and } \lim _{r \rightarrow \infty} g(r)=0 \\
& \text { 2) } \lim _{r \rightarrow \infty} r^{2} g(r)=0 .
\end{aligned}
$$

Without condition ( $7 b$ ), it can be shown that the interference power at a given terminal would be infinite for an infinite network. We will see later that in order for the characteristic function of the interference power to exist, we require that condition ( $7 \mathrm{~b}$ ) hold. The above expression for the power loss law is a far-field approximation that does not hold close to the transmitter where the transmitted signal attains a maximum. We assume that this maximum is sufficient to cause a transmission error, even in the case of one interferer; hence, the above function will result in the same probability of error while being easier to handle analytically.

Let $X$ be a Poisson process in the plane with the average number of points per unit area equal to $\lambda$. A sample function of $X$ is determined by a set of points in the plane which will correspond to locations of terminals. The probability law for $X$ is determined by (2). We assume that the probability that a terminal is transmitting is $p$. The set of transmitting terminals also forms a Poisson process $X^{t}$ with parameter $\lambda_{t}=\lambda p$. Now, with each sample function of $X^{t}$, we can associate the random variable

$$
Y=\sum g\left(r_{i}\right)
$$

where the summation is over all the points of the sample function, and $r_{i}$ is the distance of the $i$ th point to the origin. We assume that each terminal (i.e., each point of the sample function) is transmitting. Thus, $Y$ is the total interference power at the origin, and we wish to find its probability density.

Let $Y_{a}$ be the interference power received from those terminals which are in a disk or radius $a$, i.e.,

$$
Y_{a}=\sum_{r_{i} \leq a} g\left(r_{i}\right) \text {. }
$$

Thus, we have $\lim _{a \rightarrow \infty} Y_{a}=Y$. We work with $Y_{a}$ and then let $a \rightarrow \infty$ to obtain the characteristic function of $Y$. The probability density function is then the inverse Fourier transform. Let $\phi_{Y_{a}}$ be the characteristic function of $Y_{a}$, i.e.,

$$
\phi_{Y_{a}}(\omega)=\boldsymbol{E}\left(e^{i \omega Y_{a}}\right) .
$$

Using conditional expectations, this may be evaluated as

$$
\begin{aligned}
\boldsymbol{E}\left(e^{i \omega Y_{a}}\right) & =\boldsymbol{E}\left(\boldsymbol{E}\left(e^{i \omega Y_{a}} / k \text { in } \boldsymbol{D}_{a}\right)\right) \\
& =\sum_{k=0}^{\infty} \frac{e^{-\lambda_{*} \pi \iota^{2}}\left(\lambda_{l} \pi a^{2}\right)^{k}}{k !} \boldsymbol{E}\left(e^{i \omega Y_{a}} / k \text { in } \boldsymbol{D}_{a}\right)
\end{aligned}
$$

where " $k$ in $\boldsymbol{D}_{a}$ " is the event that there are $k$ terminals in the disk of radius $a$, and the expectation is over the random variable $Y_{a}$.

Now, given that there are $k$ points in $D_{a}$, and due to the nature of the Poisson process, the distribution of their locations is that of $k$ independent and identically distributed points with uniform distribution. If $R$ is the distance to the origin of a point that is uniformly distributed in $D_{a}$, then the probability density of $R$ is

$$
f_{R}(r)= \begin{cases}\frac{2 r}{a^{2}} & r \leq a \\ 0 & \text { otherwise. }\end{cases}
$$

Also, since the characteristic function of the sum of a number of independent random variables is the product of the individual characteristic functions, we have

$$
\boldsymbol{E}\left(e^{i \omega Y_{a}} / k \text { in } \boldsymbol{D}_{a}\right)=\left(\int_{0}^{a} \frac{2 r}{a^{2}} e^{i \omega g(r)} d r\right)^{k} .
$$

Note that in (13), we are considering $Y_{a}$ to be the sum of $k$ random variables which are functions of the random 
variables $R_{i}$, and each $R_{i}$ has the density given by (12). Thus, substituting (13) in (11) and summing the series, we obtain

$$
\phi_{Y_{a}}(\omega)=\exp \left(\lambda_{t} \pi a^{2}\left(\int_{0}^{a} \frac{2 r}{a^{2}} e^{i \omega g(r)} d r-1\right)\right) .
$$

Integrating by parts, the exponent of (14), letting $a \rightarrow \infty$, and using condition (7a), we obtain, after some simplification, the characteristic function of $Y$ :

$$
\phi_{Y}(\omega)=\exp \left(i \lambda_{t} \pi \omega \int_{0}^{\infty}\left[g^{-1}(t)\right]^{2} e^{i \omega t} d t\right)
$$

where $g^{-1}(\cdot)$ denotes the inverse of $g(\cdot)$.

\section{A. Statistics for a Class of Propagation Laws}

To proceed further, we must now specify $g(r)$. We specify it only up to a multiplicative constant since the results that we obtain will be independent of scaling factors. In free space, $g(r)$ would be $1 / r^{2}$; however, this does not satisfy ( $7 \mathrm{~b})$. This means, as we will see shortly, that if we are going to assume the ideal law for $g$, then we cannot assume an infinite network, for the interference power would be strongly dependent on the network size. On the ground, $g(r)$ takes the form $1 / r^{4}[17]$; thus, to work out this case and any other cases where the dependency on $r$ is not exactly an inverse fourth power, we consider the following class of propagation laws:

$$
g(r)=\frac{1}{r^{\gamma}} \quad \gamma>2 .
$$

For this class of propagation laws, (15) becomes

$$
\phi_{Y}(\omega)=\exp \left(i \lambda_{t} \pi \omega \int_{0}^{\infty} t^{-\alpha} e^{i \omega t} d t\right)
$$

where $\alpha=2 / \gamma$. The integral in (17) may be evaluated to obtain the following:

$$
\phi_{Y}(\omega)=\exp \left(-\pi \lambda_{t} \Gamma(1-\alpha) e^{-\pi \alpha / 2} \omega^{\alpha}\right) \quad \omega \geq 0
$$

where $\Gamma(\cdot)$ is the gamma function and $\phi_{Y}(\omega)=$ $\phi_{Y}^{*}(-\omega)$. The probability laws with characteristic functions given by (18) are the stable laws of exponent $\alpha$ with the restriction $0<\alpha<1$ [18]. For $\alpha=1 / 2$, (18) becomes

$$
\phi_{Y}(\omega)=\exp \left(-\pi \sqrt{\pi / 2}(1-i) \lambda_{t} \sqrt{\omega}\right) .
$$

This probability law ( $\alpha=1 / 2$ ) is the inverse Gaussian probability law, and is the only one of the stable laws, for the case $0<\alpha<1$, which is known to have a density given by a closed-form expression. The density for $\alpha=$ $1 / 2$ is given by

$$
f_{Y}(y)=\frac{\pi}{2} \lambda_{t} y^{-3 / 2} e^{-\pi^{3} \lambda_{t}^{2} / 4 y}
$$

and the distribution function is

$$
F_{Y}(y ; 1 / 2)=\operatorname{erfc}\left(\frac{\pi^{3 / 2} \lambda_{t}}{2 \sqrt{y}}\right) .
$$

In general, for $0<\alpha<1$, the densities can be found as infinite series (see [18, pp. 581-583]). Let $\rho=\pi \lambda_{t} \Gamma(1$ $-\alpha)$; then the density, obtained by taking the inverse Fourier transform of (18), is

$$
f_{Y}(y ; \alpha)=\frac{1}{\pi y} \sum_{k=1}^{\infty} \frac{\Gamma(\alpha k+1)}{k !}\left(\frac{\rho}{y^{\alpha}}\right)^{k} \sin k \pi(1-\alpha)
$$

which, for $\alpha=1 / 2$, results in a series expansion for (20). The general distribution function is

$$
F_{Y}(y ; \alpha)=\frac{1}{\pi} \sum_{k=1}^{\infty} \frac{\Gamma(\alpha k)}{k !}\left(\frac{\rho}{y^{\alpha}}\right)^{k} \sin k \pi(1-\alpha) .
$$

\section{Probability of Packet Success}

We assume a $1 / r^{4}$ propagation power loss law. Using the above distribution function for the interference, the probability of packet success is now computed. Let the distance between the transmitter and receiver be $R$. The signal power is then $1 / R^{4}$. The random variables $Y$ and $\mu$ are related through (3); hence, the probability distribution function for $\mu$ may be obtained from that of $Y$ in (21) as

$$
F_{\mu}(\mu)= \begin{cases}1-\operatorname{erfc}\left(\frac{p \lambda \pi R^{2}}{2} \sqrt{\frac{\pi}{K(\mu)}}\right) & \mu<\mu_{0} \\ 0 & \mu>\mu_{0}\end{cases}
$$

where

$$
K(\mu)=\frac{3 L}{2}\left(\frac{1}{\mu}-\frac{1}{\mu_{0}}\right) .
$$

Substituting (24) in (6), we obtain the probability of packet success

$$
P_{s}=\int_{0}^{\mu_{0}} \operatorname{erfc}\left(\frac{p \lambda \pi R^{2}}{2} \sqrt{\frac{\pi}{K(\mu)}}\right) s^{\prime}(\mu) d \mu .
$$

In the above equation, $K(\mu)+1$ may be interpreted as a multiple-access capability [14], in the case of equal interference powers, given the required SNR $\mu$ at the detector [as can be seen from (3)]. The function $s^{\prime}(\cdot)$ depends on the level of coding. For the best long codes, this function approaches a delta function at some value of $\mu$, $\mu_{c}$. The integral can then be easily evaluated, and the result corresponds to working with a reception model based on a threshold assumption. In any case, it can be seen that for the purpose of probability of packet success calculations, we can always assume a threshold model. The above equation gives the means to obtain the effective 
threshold, defined as the threshold, which when used with the threshold model, gives the same results as (25).

\section{Optimum Transmission Ranges}

We are now ready to apply the above statistics of the interference power to the determination of the optimum transmission range in a multihop network. First, we find an expression for nodal throughput as a function of the link distance $R$, the average number of terminals per unit area $\lambda$, the transmission probability $p$, and the processing gain $L$.

\section{A. Local Throughput}

The local throughput is the rate at which a terminal successfully transmits packets. For a network with uniform traffic (as we assume here) and assuming that the routing is "balanced," then the local throughput will be the same for all terminals. In terms of packets per slot, the local throughput will simply be the probability of success. Let $\zeta$ be the local throughput from terminal $\boldsymbol{A}$, and let terminal $\boldsymbol{B}$ designate a generic destination terminal; then we have

$$
\begin{aligned}
\zeta=P & {[\boldsymbol{A} \text { transmits }] \cdot P[\boldsymbol{B} \text { does not transmit }] } \\
\cdot & P[\text { packet received } / \boldsymbol{B} \text { does not transmit }] .
\end{aligned}
$$

Given that $\boldsymbol{B}$ does not transmit, it may not receive $\boldsymbol{A}$ 's transmission for one of the following two reasons: 1) $\boldsymbol{B}$ may receive a transmission from another terminal, or 2) the interference power at $\boldsymbol{B}$ may exceed the threshold. These two events are not strictly independent, and the exact calculation of the last factor of (26) is a difficult task. The lack of strict independence is due to the fact that if the interference power is large, then there is a greater probability of a large number of terminals in the vicinity of $\boldsymbol{B}$. However, we will see shortly that the probability that $\boldsymbol{B}$ receives another transmission is weakly dependent on the number of terminals in the vicinity of $\boldsymbol{B}$, and we may assume that the above two events are independent. If all terminals transmit with probability $p$ (heavy traffic case), then the first two factors of (26) are given by $p$ ( 1 $-p$ ). Now, due to the memoryless property of the Poisson distribution, if we fix a transmitter and receiver, terminals $\boldsymbol{A}$ and $\boldsymbol{B}$, the remaining terminals are still Poisson distributed with parameter $\lambda$; thus, the probability of the second of the above events is simply $P_{s}$ and (26) becomes

$$
\zeta=p(1-p) \cdot P\left[\begin{array}{l}
\boldsymbol{B} \text { chooses } \\
\boldsymbol{A} \text { 's transmission }
\end{array}\right] \cdot P_{s} .
$$

To obtain the probability that $\boldsymbol{B}$ chooses $\boldsymbol{A}$ 's transmission, we need to know how many terminals are transmitting to $\boldsymbol{B}$. If $k$ terminals (including $\boldsymbol{A}$ ) are transmitting to $\boldsymbol{B}$, then we assume that the probability that $\boldsymbol{B}$ receives $\boldsymbol{A}$ 's transmission (assuming, of course, that $\boldsymbol{B}$ does not transmit and the interference power is less than the threshold) is $1 / k$. The exact calculation that $\boldsymbol{B}$ chooses $\boldsymbol{A}$ 's transmission is very difficult. We do not know exactly how many terminals are potential transmitters to $\boldsymbol{B}$; and of the potential transmitters to $\boldsymbol{B}$, we do not know the probability of a transmission to $\boldsymbol{B}$ from a given one of them. These parameters are tied to the results that we are trying to obtain. If the number of potential transmitters to $B$ is $n$, and if we assume that each of these can transmit to $n$ terminals (hence, the probability of a transmission to $B$ is $p / n$ ), i.e., local traffic is uniform, then we have

$$
\begin{aligned}
& P\left[\begin{array}{l}
\boldsymbol{B} \text { chooses } \\
\boldsymbol{A}
\end{array}\right] \\
& \quad=\sum_{i=0}^{n-1}\left(\begin{array}{l}
n-1 \\
i
\end{array}\right)\left(\frac{p}{n}\right)^{i}\left(1-\frac{p}{n}\right)^{n-1-i} \cdot \frac{1}{i+1} \\
& \quad=\frac{1}{p}\left(1-\left(1-\frac{p}{n}\right)^{n}\right)
\end{aligned}
$$

where the summation results from conditioning on the number of transmissions addressed to $\boldsymbol{B}$. Substituting (28) in (27), we obtain

$$
\zeta=(1-p)\left(1-\left(1-\frac{p}{n}\right)^{n}\right) \cdot P_{s} \triangleq \tau_{n}(p) \cdot P_{s} .
$$

We have plotted the factor $\tau_{n}(p)$ versus $p$ for different values of $n$ in Fig. 2. From the plots, we see that for $n>$ $2, \tau_{n}(p)$ is not too sensitive to $n$, and we verify our independence assumption. We therefore assume that $n=$ $\infty$. Letting $n \rightarrow \infty$ in (29), we obtain

$$
\zeta=\tau(p) \cdot P_{s}
$$

where

$$
\tau(p) \triangleq \tau_{\infty}(p)=(1-p)\left(1-e^{-p}\right) .
$$

In [19], we discussed two factors which affect the throughput of a spread spectrum network and referred to them as the tendency to pair up and the availability of a channel. In terms of our notation here, $\tau$ is the tendency to pair up (given per terminal) and $P_{s}$ is the availability of a channel. From the above assumption that the two events given by 1) and 2) are independent, we have gained the factorization of the throughput into these two factors.

\section{B. Expected Progress per Slot}

Using the system model previously described, we determine the optimum transmission range by using the expected forward progress of a packet per slot as the performance criterion. In a multihop network, the probability of packet success increases as the link distance decreases. However, in choosing a small link distance, the number of hops that the packet must take is increased and the network internal traffic is artificially increased. This, in turn, causes the probability of packet success to decrease. A performance measure is required which increases with an increase in the packet success probability and decreases as the number of hops increases. The expected forward 


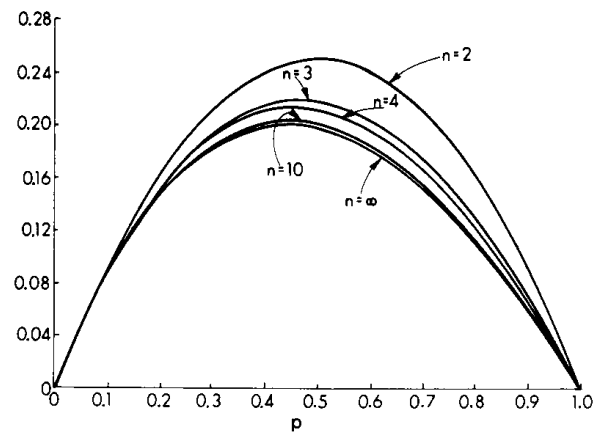

Fig. 2. Tendency to pair up $\tau_{n}(p)$ versus the probability of transmission $p$.

progress per slot [1]-[5] is such a measure. The expected forward progress per slot $Z$ is

$$
Z=\zeta \cdot R \text {. }
$$

To express the following results in terms of dimensionless quantities, we let $N=\lambda \pi R^{2}$. $N$ will be the average number of terminals which are closer to $\boldsymbol{A}$ than $\boldsymbol{B}$, and in terms of $N, R=\sqrt{N / \pi \lambda}$. For the propagation law given by $\gamma$ $=4$ (i.e., $\alpha=1 / 2$ ), we obtain the following [it results from substituting (30) in (31) and using (28) and (25)]:

$$
\begin{aligned}
\sqrt{\lambda} Z= & \sqrt{N / \pi}(1-p)\left(1-e^{-p}\right) \\
& \cdot \int_{0}^{\mu_{0}} \operatorname{erfc}\left(\frac{p N}{2} \sqrt{\frac{\pi}{K(\mu)}}\right) s^{\prime}(\mu) d \mu .
\end{aligned}
$$

We have multiplied $Z$ by $\sqrt{\lambda}$ in (32) so that the right-hand side is dimensionless. Note that contrary to the analysis in [1]-[5], $N$ is not to be interpreted as the average number of neighbors of a given terminal ( $\boldsymbol{A}$ in our case). Rather, $N$ is the average number of terminals that are within the chosen range.

To compute (32), we need the coding function $s(\mu)$. As an example, we assume a highly coded system so that $s(\cdot)$ is close to a step function at some critical SNR which we denote as the parameter $\mu_{c}$. The derivative is then approximately given by $s^{\prime}(\mu)=\delta\left(\mu-\mu_{c}\right)$ and (32) becomes

$\sqrt{\lambda} Z=\sqrt{N / \pi}(1-p)\left(1-e^{-p}\right) \operatorname{erfc}\left(\frac{p N}{2} \sqrt{\frac{\pi}{K\left(\mu_{c}\right)}}\right)$.

In the above, $K$ is a function of $\mu_{c}$, the required SNR for the coding scheme used, and also implicitly a function of $\mu_{0}$ and the processing gain $L . K$ is directly proportional to the processing gain. The parameter $\mu_{c}$ is mainly dependent on the level of coding, and assuming a fixed background noise level, $\mu_{0}$ is mainly dependent on the link distance $R$. Setting $K$ equal to zero, we obtain the maximum link distance as $R_{0}=\left(T_{s} /\left(\mu_{c} N_{0}\right)\right)^{1 / 4}$. Even though the maximum link distance is $R_{0}$, the probability of re-

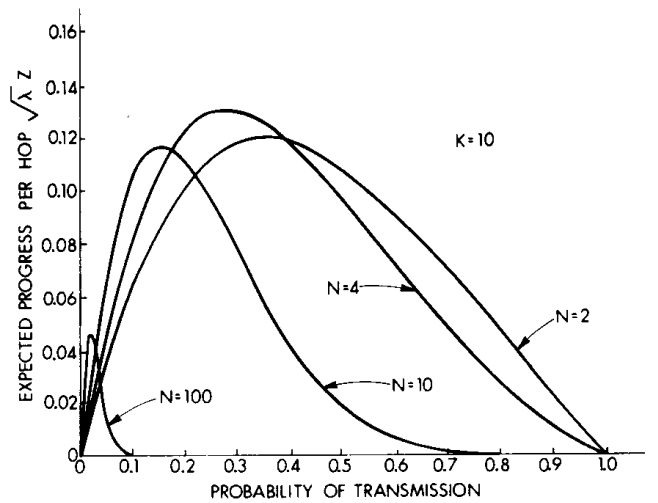

Fig. 3. Expected progress per hop versus probability of transmission.

ception becomes very small for link distances close to $R_{0}$, and an optimum transmission range will be considerably smaller than $R_{0}$.

\section{Parameter Optimization}

We have plotted (33) in Figs. 3-5 for the cases of $K\left(\mu_{c}\right)$ equal to 10,100 , and 1000 and for different values of the parameter $N$. From the figures, we note that in each case, there is an optimum value of $N$, and as $N$ increases from this value, the expected forward progress decreases. Although we only show four plots for each case of $K$, the $N$ yielding the maximum expected progress for the given $K$ and at the optimum $p$ has been chosen as the value over all $N$ which yields the maximum expected progress at its optimum probability of transmission $p$.

In Fig. 6, we show plots of the maximum expected progress versus $N$ with $K$ as a parameter, that is, the peaks in the plots of Figs. 3-5 versus $N$. An interesting observation is the relation between the optimum $N$, that is, the $N$ yielding the maximum expected progress in Fig. 6, and the corresponding value of $K$.

To find the parameters yielding the maximum expected forward progress, we may optimize (32) over the parameters $p$ and $N$. Setting the partial derivatives with respect to $p$ and $N$ to zero yields the following two equations:

$$
\begin{gathered}
\int_{0}^{\mu_{0}}\left(\frac{4 \psi(\mu)}{\sqrt{\pi}} e^{-\psi(\mu)^{2}}-\operatorname{erfc}[\psi(\mu)]\right) s^{\prime}(\mu) d \mu=0 \\
\int_{0}^{\mu_{0}} \frac{e^{\psi(\mu)^{2}}}{\psi(\mu)} \operatorname{erfc}[\psi(\mu)] s^{\prime}(\mu) d \mu \\
=\frac{2(1-p)\left(1-e^{-p}\right)}{\sqrt{\pi} p\left[e^{-p}(2-p)-1\right]}
\end{gathered}
$$

where $\psi(\mu)=(p N / 2) \sqrt{\pi / K(\mu)}$. To solve the above, we assume a fixed $L$ and $\mu_{0}$, and then solve (34a) for the function $\psi(\mu)$. For a fixed $L$ and $\mu_{0}$, such a solution amounts to finding a value for the product $p N$. We then 


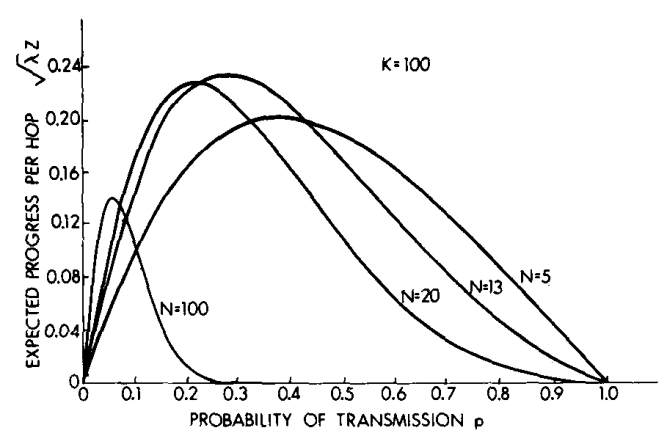

Fig. 4. Expected progress per hop versus probability of transmission.

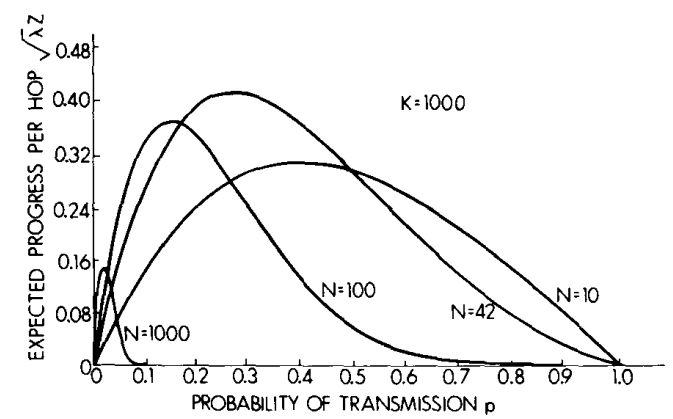

Fig. 5. Expected progress per hop versus probability of transmission.

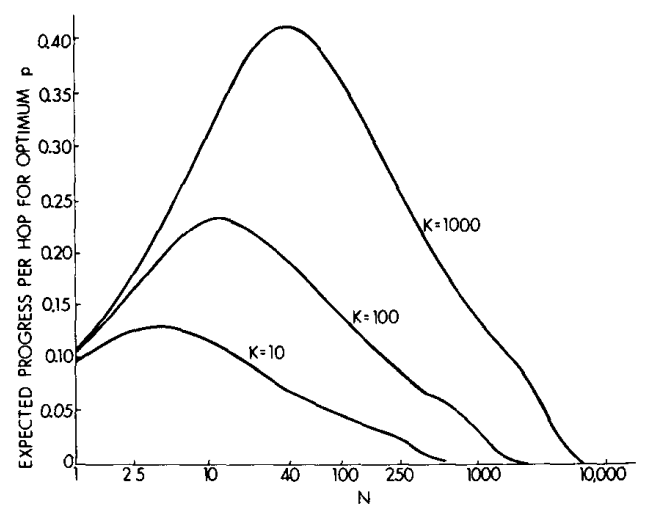

Fig. 6. Expected progress per hop for optimum probability of transmission versus the expected number of interferers that are closer to the receiver than the transmission $N$.

substitute for $\psi(\mu)$ in the left side of (34b), evaluate the integral, and solve the resulting equation for $p . N$ is then obtained from $\psi$ and $p$. As an example, for $s(\cdot)$ equal to a step function at $\mu=\mu_{c}$, we obtain

$$
N_{0}=1.33 \sqrt{K\left(\mu_{c}\right)}
$$

and

$$
p_{0}=0.271 \text {. }
$$

\section{Generalizations}

In the previous analysis, we were concerned with a network on the plane, and we determined optimum transmission ranges only for the case of an inverse fourth power propagation loss law. It turns out that the results obtained for the interference statistics can be easily generalized to $d$-dimensional space, and similar results for the optimum transmission range can be obtained. Although only the additional cases of one dimensional and three dimensional have any physical significance, the following expressions hold for any dimension.

\section{A. Interference Statistics}

We assume, as previously, that we have an infinite network. The terminals are distributed according to a Poisson law on a $d$-dimensional space. The meaning here is that if $V$ is the volume of a region $\boldsymbol{R}$ in $d$-dimensional space, then the number of terminals in $\boldsymbol{R}$ has the following distribution:

$$
P[k \text { in } R]=\frac{e^{-\lambda V}(\lambda V)^{k}}{k !}
$$

where $\lambda$ is the average number of terminals per unit volume.

We may follow the same procedure that led to (15) where, instead of working with a sphere in two-dimensional space (i.e., a circle), we work with a sphere in $d$-dimensional space. The generalized version of (15) is

$$
\phi_{Y}(\omega)=\exp \left(i K_{d} \lambda \omega \int_{0}^{\infty}\left[g^{-1}(t)\right]^{d} e^{i \omega t} d t\right)
$$

where $K_{d}$ is the volume of the unit sphere in $d$-dimensional space $\left(K_{1}=2, K_{2}=\pi, K_{3}=4 \pi / 3\right.$, etc.). Assuming the class of propagation laws given by (16), (37) becomes

$$
\phi_{Y}(\omega)=\exp \left(-K_{d} \lambda \Gamma(1-\alpha) e^{-i \pi \alpha / 2} \omega^{a}\right) \quad \omega>0
$$

where $\alpha=a / \gamma$. We note that for the interference to be finite, we must have $\gamma>d$.

The probability distribution function in the general case is given by (23) where $\rho=K_{d} \lambda \Gamma(1-\alpha)$.

\section{B. Optimum Transmission Ranges}

In a similar manner as for (32), we may calculate the expected forward progress and obtain

$$
\begin{aligned}
(\lambda)^{1 / d} Z= & \left(N / K_{d}\right)^{1 / d}(1-p)\left(1-e^{-p}\right) \\
& \cdot\left(1-\frac{1}{\pi} \sum_{n=1}^{\infty} \frac{\Gamma(\alpha n)}{n !} \sin n \pi(1-\alpha)\right. \\
& \left.\cdot \int_{0}^{\mu_{0}}\left(\frac{p N \Gamma(1-\alpha)}{K(\mu)^{\alpha}}\right)^{n} s^{\prime}(\mu) d \mu\right) .
\end{aligned}
$$

As previously, $Z$ is multiplied by $\lambda^{1 / d}$ so as to make the expected forward progress dimensionless. Also, as pre- 
TABLE I

SOME OF THE CONSTANTS IN (40)

\begin{tabular}{|c|c|c|c|c|c|}
\hline \hline \multicolumn{6}{|c|}{$C(d, \alpha)$} \\
\hline$d \alpha$ & $1 / 4$ & $1 / 3$ & $1 / 2$ & $2 / 3$ & $3 / 4$ \\
\hline 2 & 1.73 & 1.62 & 1.34 & 0.94 & 0.71 \\
\hline 3 & 0.90 & 0.88 & 0.77 & 0.58 & 0.46 \\
\hline
\end{tabular}

viously, we would like to find the optimum $N$ as a function of $K$ and for the optimum transmission probability $p$. We may optimize over the parameters $p$ and $N$ as before. For the case of a step coding function $s(\cdot)$, we summarize the results as follows: for a given $d$ and $\alpha$, the optimum transmission range $N_{0}$ is given by the relation

$$
N_{0}(d, \alpha)=C(d, \alpha) K^{\alpha}\left(\mu_{c}\right) .
$$

As previously, $N_{0}$ is proportional to a power of the parameter $K\left(\mu_{c}\right)$. We give a few values of the constant $C$ in Table I.

We summarize the above results. In obtaining the interference statistics, the important parameters are $d$, the dimension of the network, and $\gamma$, the exponent of the propagation loss law. Given these two parameters, we define $\alpha=d / \gamma$. The interference statistics are then given by the stable law of exponent $\alpha$, and the optimum $N_{0}$ is proportional to $K^{\alpha}\left(\mu_{c}\right)$ where $K(\cdot)$ is defined as in (24) and Table I gives a few of the proportionality constants.

\section{Summary and Conclusions}

Multihop packet radio models used in the past have used the concept of a transmission radius where within a given radius of a transmitter, a packet has an "equal" probability of being received. This model essentially assumes a step function for the signal strength versus distance from the transmitter. In this paper, we have taken a new approach: a signal is assumed to decay in strength according to a gradually decreasing function of the distance from the transmitter. By assuming a random distribution of the terminals, we were able to obtain the statistics of the interference power from all other transmissions at a particular receiver. Assuming an inverse power law for the signal strength versus distance from the transmitter, we showed that the probability laws of the interference power are the stable laws with parameter $\alpha$ restricted to $0<\alpha<1$. The case of an inverse fourth power propagation law, which results from ground wave propagation, corresponds to the stable law with $\alpha=1 / 2$, which has a density known as the inverse Gaussian probability density.

In a multihop packet radio network, there is usually a tradeoff between the distance covered in one hop and the probability of a successful transmission. Using the above interference analysis, we proceeded to obtain the optimum transmission range for a DSSS network. For a narrow-band packet radio network, previous results have concluded that the range of a transmission should be such that on the average there are approximately six-eight ter- minals closer to the transmitter than the receiver. For a spread spectrum system, we expect different results since multiple simultaneously successful transmissions are possible. In the spirit of the analysis of Kleinrock and Silvester [1]-[3], we have concluded that in a direct sequence spread spectrum network, the range of a transmission should be chosen so that on the average there are 1.33 $\sqrt{K\left(\mu_{c}\right)}$ terminals closer to the transmitter than the receiver where $K\left(\mu_{c}\right)$ is a parameter that is proportional to the processing gain, which can be interpreted as the effective maximum number of simultaneously successful transmissions possible in some region as a function of the effective SNR $\mu_{i}$ required for a particular coding scheme.

\section{REFERENCES}

111 L. Kleinrock and J. A. Silvester, "Optimum transmission radii for packet radio networks or why six is a magic number, " in Conf. Rec. Nat. Telecommun. Conf. . Dec. 1978, pp. 4.3.1-4.3.5.

[2] J. A. Silvester, "On the spatial capacity of packet radio networks," Dep. Comput. Sci., Univ. California, Los Angeles. Eng. Rep. UCLAENG-8021, May 1980 .

[3] L. Kleinrock, and J. A. Silvester, "Spatial reuse in multihop packet radio networks," Proc. IEEE, vol. 75, pp. 116-134, Jan. 1987.

[4] H. Takagi and L. Kleinrock. "Optimal transmission ranges for randomly distributed packet radio terminals," IEEE Trans. Commun. . vol. COM-32, pp. 264-257, Mar. 1984.

[5] T. C. Hou and V. O. K. Li, " Transmission range control in multihop packet radio networks," IEEE Trans. Commun., vol. COM-34, pp. 38-44, Jan. 1986.

[6] M. K. Simon, J. K. Omura, R. A. Scholtz, and B. K. Levitt, Spread Spectrum Communications, Vol. I. Rockville, MD: Computer Science Press, 1984

17] W. C. Peng, "Some communication jamming games," Ph.D. dissertation, Dep. Elec. Eng., Univ. Southern California, Los Angeles. Jan. 1986.

18] L. G. Roberts, "ALOHA packet system with and without slots and capture," ARPA Network Inform. Cen., Stanford Res. Inst. Menlo Park, CA, ASS Note 8 (NIC 11290), June 1972; reprinted in Comput. Commun. Rev., vol. 5, pp. 28-42, Apr. 1975.

[9] L. Fratta and D. Sant, "Some models of packet radio networks with capture," in Proc. Int. Conf. Comput. Commun. Oct. 1980, pp. 155161

[10] R. Nelson and L. Kleinrock, "The spatial capacity of a slotted ALOHA multihop packet radio network with capture," IEEE Trans. Commun., vol. COM-32, pp. 684-694. June 1984.

[11] E. S. Sousa and J. A. Silvester, "Determination of optimum transmission ranges in a multi-hop spread spectrum network," in Proc. MILCOM. Boston. MA. Oct. 1985, pp. 449-454.

[12] G. R. Cooper and R. W. Nett]eton, "A spread-spectrum technique for high capacity mobile communications," IEEE Trans. Vehic. Technol., vol. VT-27, Nov. 1978.

[13] S. A. Musa and W. Wasylkiwskyj. "Co-channel inference of spread spectrum systems in a multiple user environment, "IEEE Trans. Commun. vol. COM-26, pp. 1405-1413, Oct. 1978.

[14] C. L. Weber, G. K. Huth, and B. H. Batson. "Performance consideration of code division multiple-access systems," IEEE Trans. Vehic. Technol., vol. VT-30, pp. 3-9. Feb. 1981

[15] O. DeSouza, P. Sen, and R. R. Boorstyn, "Performance analysis of spread spectrum packet radio networks," in Proc. MILCOM, San Diego, CA. Oct. 1988, pp. 599-604.

[16] M. B. Pursley, "Performance evaluation for phase-coded spreadspectrum multiple-access communication-Part I: System analysis," IEEE Trans. Commun., vol. COM-25 pp. 795-799, Aug. 1977.

[17] J. J. Egli, "Radio propagation above 40 Mc over irregular terrain," Proc. IRE. pp. 1383-1391, Oct. 1957.

[18] W. Feller, An Introduction to Probability Theory and Its Applications, Vol. II. Wiley, 1966, pp. 554-583.

[19] E. S. Sousa and J. A. Silvester, "Spreading code protocols for distributed spread spectrum packet radio networks," IEEE Trans. Com mun. . vol. 36, pp. 272-281, Mar. 1988. 


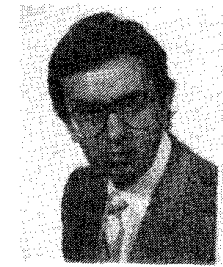

Elvino S. Sousa ( $S^{\prime} 80-M^{\prime} 86$ ) was born in the Azores, Portugal, on December 28,1956 . He received the B.A.Sc. degree in engineering science and the M.A.Sc. degree in electrical engineering from the University of Toronto, Toronto, Ont., Canada, in 1980 and 1982 , respectively, and the $\mathrm{Ph} . \mathrm{D}$. degree in electrical engineering from the University of Southern California degree in 1985.

He was a Teaching Assistant at the University of Toronto, a Research Assistant at the University of Southern California, and is currently with the Department of Electrical Engineering, University of Toronto. He has performed research in the areas of data transmission and spread spectrum packet radio networks. He is also interested in optical communications and the performance of distributed computer systems, on which he has consulted for Technology Transfer Institute. In the past three years at the University of Toronto, he has taught graduate courses in error-correcting codes and mobile communications.

Dr. Sousa is a member of Etta Kappa Nu and the Association of Professional Engineers of Ontario. He has held a Natural Sciences and Engineering Research Council of Canada (NSERC) Postgraduate Scholarship, was a recipient of the IEEE Communications Society Student Scholarship, and is currently an NSERC University Research Fellow.

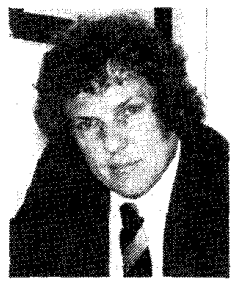

John A. Silvester (M'79-SM'85) was born in Kent, England, in 1950. He received the B.A. and M.A. degrees in mathematics and operations re search from the University of Cambridge, Cam bridge, England, in 1971 and 1975 , the M.S. degree in statistics and computer science from West Virginia University, Morgantown, in 1973, and the Ph.D. degree in computer science from the University of California, Los Angeles, in 1980.

Since 1979 he has been at the University of Southern California, Los Angeles, where he is an
Associate Professor in the Department of Electrical Engineering and $\mathrm{Di}$ rector of the Computer Engineering Division. He teaches courses in com puter networks, queueing theory, computer system performance evaluation, and computer architecture. His current research interests are the performance evaluation and modeling of computer communication networks and distributed systems, packet radio and spread spectrum radio networks, high-speed networks, local area networks, and multiple-access protocols. He is the author of over 60 technical papers and has lectured both in the United States and abroad. He has consulted for many of the leading networking companies, the U.S. Army, and was Director of Consulting for Technology Transfer Institute from 1984 to 1986 where he is still a leading consultant. $\mathrm{He}$ also teaches short courses on packet radio, network design tools, and data communications fundamentals.

Dr. Silvester is a member of the ACM and has been active in the Technical Committee on Computer Communications of the IEEE Communications Society since 1981, acting as Conference Coordinator from 1983 to 1985 and Chairman from 1985 to 1987 . He was Program Chairman of the First IEEE Computer Communications Workshop (1986), is on the INFOCOM conference board, was Vice Chairman of INFOCOM' 89 , and General Chair for INFOCOM 90 . He has served on the Program Committees of INFOCOM, the Data Communications Symposium, and the International Conference on Computer Communications. 